MPP-2006-33

\title{
On Electroweak Matching Conditions for Top Pair Production at Threshold
}

\author{
André H. Hoang and Christoph J. Reißer \\ Max-Planck-Institut für Physik \\ (Werner-Heisenberg-Institut), \\ Föhringer Ring 6, \\ 80805 München, Germany*
}

\begin{abstract}
We determine the real parts of electroweak matching conditions relevant for top quark pair production close to threshold in $e^{+} e^{-}$annihilation at next-to-next-to-leading logarithmic (NNLL) order. Numerically the corrections are comparable to the NNLL QCD corrections.
\end{abstract}

*Electronic address: ahoang@mppmu.mpg.de, reisser@mppmu.mpg.de 


\section{INTRODUCTION}

The detailed study of top quark pair production close to threshold constitutes a major part of the top quark physics program at the International Linear Collider (ILC) during its first phase running at lower energies. While the top quark mass (in a threshold mass scheme [1, 2, 3] ) can be measured with a precision of around $100 \mathrm{MeV}$ from the c.m. energy where the cross section line-shape rises, accurate measurements of the top quark total width, the strong coupling and (in the case of a light Higgs) the top Yukawa coupling can be gained from the normalization and the form of the line-shape [4]. Electroweak effects play a crucial role for theoretical predictions of top pair threshold production. Apart from the $t \bar{t}$ production process, mediated either by virtual photon or $\mathrm{Z}$ boson exchange, one also needs to account for initial state QED beam effects and the finite top quark lifetime in the leading order approximation. On the other hand, due to the fact that, close to threshold, the top quarks are produced with small relative velocities, $v \ll 1$, singularities proportional to $\left(\alpha_{s} / v\right)^{n}$ and $\left(\alpha_{s} \ln v\right)^{n}$ arise from virtual and real gluon radiation in $\mathcal{O}\left(\alpha_{s}^{n}\right)$ QCD diagrams that need to be summed up to all orders in $\alpha_{s}$. These singularities arise from ratios of the kinematical scales $m_{t}$ ("hard"), $p_{t} \sim m_{t} v \sim m_{t} \alpha_{s}$ ("soft") and $E_{t} \sim m_{t} v^{2} \sim m_{t} \alpha_{s}^{2}$ ("ultrasoft") that are governing the nonrelativistic $t \bar{t}$ dynamics, where $p_{t}$ and $E_{t}$ are the top three-momentum and kinetic energy, respectively. Since the Standard Model top quark width $\Gamma_{t} \approx \Gamma_{t}(t \rightarrow b W) \approx 1.5 \mathrm{GeV}$ is numerically comparable to the average top kinetic energy, it serves as an infrared cutoff and protects the nonrelativistic $t \bar{t}$ dynamics from nonperturbative QCD effects.

In recent years most work in the literature on top pair threshold production was focused on determining higher order QCD corrections using nonrelativistic QCD (NRQCD). ${ }^{1}$ In the fixed-order approach, where the singularities $\propto\left(\alpha_{s} / v\right)^{n}$ are systematically summed, nextto-next-to-leading order (NNLO) corrections to the total cross section are fully known [1] and even some NNNLO analyses have become available recently (see e.g. Refs. [5, 6] ). In the renormalization group improved approach both singularities $\propto\left(\alpha_{s} / v\right)^{n}$ and $\propto\left(\alpha_{s} \ln v\right)^{n}$ are systematically summed. Here, the full set of NNLL order corrections is known except for the full NNLL running of the dominant S-wave $t \bar{t}$ production current [7, 8, 9]. At present the theoretical QCD normalization uncertainties of the NNLO fixed-order prediction for the total $t \bar{t}$ cross section close to threshold is estimated to be of the order $20 \%$ [1], while for the (yet incomplete) NNLL order renormalization group improved prediction it is 6\% [10].

As far as electroweak corrections are concerned no complete determination of all effects beyond the leading order level has yet been achieved for the total cross section. At leading order the three electroweak effects mentioned above can be treated independently: The top pair production is described by $\left(e^{+} e^{-}\right)(t \bar{t})$ effective NRQCD operators with Wilson coefficients that account for the propagation of the virtual photon and the $\mathrm{Z}$ boson mediating the $t \bar{t}$ production process. The QED beam effects consisting of the machine-dependent beam energy spread, beamstrahlung and initial state radiation can be conveniently implemented through a luminosity spectrum that is convolved into the theoretical predictions without any initial state QED corrections [4, 11, 12]. The top quark width, on the other hand, is incorporated into NRQCD by an imaginary matching condition of a bilinear top quark mass operator and can be effectively accounted for by shifting the c.m. energy into the complex

\footnotetext{
${ }^{1}$ We use the term NRQCD to refer to a generic low-energy effective theory which describes nonrelativistic $t \bar{t}$ pairs and bound state effects and not for a theory valid only for scales $m_{t}>\mu>m_{t} v$.
} 
plane, $\sqrt{s} \rightarrow \sqrt{s}+i \Gamma_{t}$ [13, 14]. In Ref. [15] a systematic procedure for the theoretical treatment of the effects from the finite top quark lifetime for the total cross section beyond leading logarithmic (LL) order in the renormalization group improved approach was presented. It was shown that the effects of the top decay can be systematically incorporated when NRQCD is matched to the Standard Model by including the absorptive parts in electroweak matching conditions that are related to the top quark decay. A systematic expansion scheme can be achieved by using the power counting [15, 16]

$$
v \sim \alpha_{s} \sim g \sim g^{\prime}
$$

for $\alpha_{s}$ and the $\mathrm{SU}(2)$ and $\mathrm{U}(1)$ gauge couplings $g$ and $g^{\prime}$. The parametric counting scheme in Eq. (II) is motivated by the close numeric relation between the average top quark kinetic energy and the top quark width, $E_{t} \sim m_{t} \alpha_{s}^{2} \approx \Gamma_{t} \sim m_{t} \alpha$. It shows that one-loop electroweak corrections to the $t \bar{t}$ production process can contribute at NNLL order. In particular, it was shown in Ref. [15] that, due to gauge cancellations, there are no electroweak matching corrections to the NRQCD potential operators and heavy-quark-gluon interactions up to NNLL order. However, nontrivial (real and imaginary) electroweak matching conditions exist for the dominant $\left(e^{+} e^{-}\right)(t \bar{t})$ operators as well as for the top bilinear operators due to higher order corrections to the top quark width and the relativistic time dilatation effects. In Ref. [15] the imaginary parts of these electroweak matching conditions were analyzed in detail. It was found that they lead to corrections of the line-shape form between $2 \%$ and $10 \%$ and can shift the $1 \mathrm{~S}$ peak position by up to $50 \mathrm{MeV}$.

In this work we analyze the real parts of the NNLL electroweak matching conditions of the dominant S-wave $\left(e^{+} e^{-}\right)(t \bar{t})$ operators. We exclude pure QED corrections ${ }^{2}$ from our considerations. A complete treatment of pure QED effects together with a consistent computation of NNLL initial state (beam) and final state corrections shall be postponed to subsequent work. The corrections considered in this work have been discussed in detail before in Ref. [17] and were partly reanalyzed again later in Ref. [18]. Here we present the results of a new independent computation and point out a number of discrepancies to the results in these references.

The program of this paper is as follows. In Sec.II we explain the notation relevant for the matching conditions of the $\left(e^{+} e^{-}\right)(t \bar{t})$ operators considered in this work. In Sec.III we present our results and discuss the differences to Refs. [17] and [18]. Section [IV contains a brief numerical analysis, and in Sec. $\nabla$ we conclude.

\section{NOTATION}

In this section we set up the notation for the electroweak matching conditions to the dominant S-wave $t \bar{t}$ production and annihilation operators relevant for $e^{+} e^{-}$collisions. To be specific we use the notations that were introduced in Ref. [15] in the framework of vNRQCD [19, 20]. The dominant effective theory operators to be used for $t \bar{t}$ pair production

\footnotetext{
${ }^{2}$ In this context pure QED corrections correspond to loop diagrams that contain a photon but no other Standard Model gauge bosons.
} 
in an S-wave spin triplet state have the form

$$
\begin{aligned}
\mathcal{O}_{V, \boldsymbol{p}} & =\left[\bar{e} \gamma_{j} e\right] \mathcal{O}_{\boldsymbol{p}, 1}^{j}, \\
\mathcal{O}_{A, \boldsymbol{p}} & =\left[\bar{e} \gamma_{j} \gamma_{5} e\right] \mathcal{O}_{\boldsymbol{p}, 1}^{j},
\end{aligned}
$$

where

$$
\mathcal{O}_{\boldsymbol{p}, 1}^{j}=\left[\psi_{\boldsymbol{p}}^{\dagger} \sigma_{j}\left(i \sigma_{2}\right) \chi_{-\boldsymbol{p}}^{*}\right]
$$

and the indices $j=1,2,3$ are summed. They give the contribution $\Delta \mathcal{L}=$ $\sum_{\boldsymbol{p}}\left(C_{V}(\nu) \mathcal{O}_{V, \boldsymbol{p}}+C_{A}(\nu) \mathcal{O}_{A, \boldsymbol{p}}\right)+$ H.c. to the effective theory Lagrangian where the hermitian conjugation is referring to the operators only and does, due to unitarity, not act on the complex Wilson coefficients [15]. The term $\nu$ is the dimensionless vNRQCD renormalization group parameter, where $\nu=1$ corresponds to the hard matching scale $\left(\mu=m_{t}\right)$ and $\nu \sim \alpha_{s}$ indicates the range of scales where the effective theory matrix elements are evaluated. The relation between the Wilson coefficients at the matching scale $\nu=1$ and for $\nu<1$ has the multiplicative form $C_{V, A}(\nu) / C_{V, A}(1)=\exp \left(f\left(m_{t}, \nu\right)\right)$. The function $f$ is fully known at NLL order 20, 21]. At NNLL order only the nonmixing contributions to the anomalous dimensions [9] and the subleading mixing effects from the spin-dependent potentials [22] are presently known. The matching conditions for the Wilson coefficients at NNLL order (and without accounting for pure QED corrections) can be written in the form $(i=V, A)$

$$
C_{i}(\nu=1)=C_{i}^{\mathrm{born}}(\nu=1)\left(1-\frac{2 C_{F} \alpha_{s}\left(m_{t}\right)}{\pi}+\left(\frac{\alpha_{s}\left(m_{t}\right)}{\pi}\right)^{2} a^{(2)}\right)+i C_{i}^{\mathrm{bW}, \mathrm{abs}}+C_{i}^{\mathrm{ew}} .
$$

The terms $C_{V, A}^{\text {born }}$ refer to the tree level matching conditions originating from virtual photon and $\mathrm{Z}$ boson exchange and read

$$
\begin{aligned}
& C_{V}^{\text {born }}(\nu=1)=\frac{\alpha \pi}{m_{t}^{2}\left(4 c_{w}^{2}-x\right)}\left[Q_{e} Q_{t}(4-x)+Q_{t}-Q_{e}-\frac{1}{4 s_{w}^{2}}\right], \\
& C_{A}^{\text {born }}(\nu=1)=-\frac{\alpha \pi}{m_{t}^{2}\left(4 c_{w}^{2}-x\right)}\left[Q_{t}-\frac{1}{4 s_{w}^{2}}\right],
\end{aligned}
$$

where

$$
x \equiv \frac{M_{W}^{2}}{m_{t}^{2}},
$$

$Q_{f}$ is the fermion electric charge, $s_{w}\left(c_{w}\right)$ the sine (cosine) of the weak mixing angle and $\alpha$ is the electromagnetic coupling. The factors multiplying the tree level contributions represent the hard QCD matching conditions which are presently known up to order $\alpha_{s}^{2}$. The term $C_{V, A}^{\mathrm{bW} \text {,abs }}$ in Eq. (4) describes the imaginary (absorptive) parts of the matching conditions. They arise from cuts in the one-loop electroweak diagrams that are related to intermediate states that arise in the top decay and were identified in Ref. 15]. In predictions for the total cross section they account for interference contributions of the amplitude for the double resonant signal process $e^{+} e^{-} \rightarrow t \bar{t} \rightarrow b W^{+} \bar{b} W^{-}$with the single resonant amplitudes describing the processes $e^{+} e^{-} \rightarrow t+\bar{b} W^{-} \rightarrow b W^{+} \bar{b} W^{-}$and $e^{+} e^{-} \rightarrow b W^{+} \bar{t} \rightarrow b W^{+} \bar{b} W^{-}$. According to the power counting scheme in Eq. (11) these interference effects contribute at NNLL order to the NRQCD matrix elements for the total cross section. In Ref. [15] it was 
also shown that these NNLL matrix elements contain phase space UV-divergences that lead to the NLL running of $\left(e^{+} e^{-}\right)\left(e^{+} e^{-}\right)$forward scattering operators. Finally, the terms $C_{V, A}^{\mathrm{ew}}$ in Eq. (41) refer to the real parts of the NNLL (one-loop) electroweak matching corrections, which are the main focus of this work.

Using the optical theorem the contribution of the effective theory operators in Eqs. (2) to the total $t \bar{t}$ production cross section close to threshold reads

$$
\sigma_{\text {tot }} \sim \frac{1}{s} \operatorname{Im}\left[\left(C_{V}^{2}(\nu)+C_{A}^{2}(\nu)\right) L^{l k} \mathcal{A}_{1}^{l k}\right]
$$

where $\left(k+k^{\prime}=(\sqrt{s}, 0)\right.$ and $\left.\hat{\mathbf{e}}=\mathbf{k} /|\mathbf{k}|\right)$

$$
\begin{aligned}
L^{l k} & =\frac{1}{4} \sum_{e^{ \pm} \text {spins }}\left[\bar{v}_{e^{+}}\left(k^{\prime}\right) \gamma^{l}\left(\gamma_{5}\right) u_{e^{-}}(k)\right]\left[\bar{u}_{e^{-}}(k) \gamma^{k}\left(\gamma_{5}\right) v_{e^{+}}\left(k^{\prime}\right)\right] \\
& =\frac{1}{2}\left(k+k^{\prime}\right)^{2}\left(\delta^{l k}-\hat{e}^{l} \hat{e}^{k}\right)
\end{aligned}
$$

is the spin- and angular-averaged lepton tensor and $\left(\hat{q} \equiv\left(\sqrt{s}-2 m_{t}, 0\right)\right)$

$$
\mathcal{A}_{1}^{l k}=i \sum_{\boldsymbol{p}, \boldsymbol{p}^{\prime}} \int d^{4} x e^{-i \hat{q} \cdot x}\left\langle 0\left|T \mathcal{O}_{\boldsymbol{p}, 1}^{l \dagger}(0) \mathcal{O}_{\boldsymbol{p}^{\prime}, 1}^{k}(x)\right| 0\right\rangle
$$

is the time ordered product of the $t \bar{t}$ production and annihilation operators $\mathcal{O}_{\boldsymbol{p}, 1}^{j}$ and $\mathcal{O}_{\boldsymbol{p}, 1}^{j \dagger}$, which describes the nonrelativistic dynamical effects. In the case of nonzero electron or positron polarization the formula in Eq. (8) is simply modified by

$$
C_{V}^{2}+C_{A}^{2} \stackrel{P_{+}, P_{-} \neq 0}{\longrightarrow}\left(1-P_{+} P_{-}\right)\left(C_{V}^{2}+C_{A}^{2}\right)+\left(P_{-}-P_{+}\right) 2 C_{V} C_{A}
$$

where $P_{\mp}$ refers to electron/positron polarization along the momentum direction. Since $C_{V}$ and $C_{A}$ have opposite signs (see Tab. I) the cross section is large for positive $e^{+}$and negative $e^{-}$polarization. In the unpolarized case the relative NNLL corrections to the total cross section coming from the real parts of the one-loop electroweak matching conditions read

$$
\Delta^{\mathrm{ew}}=\frac{\delta \sigma_{\mathrm{tot}}^{\mathrm{ew}}}{\sigma_{\mathrm{tot}}}=\frac{2 C_{V}^{\mathrm{born}} C_{V}^{\mathrm{ew}}+2 C_{A}^{\mathrm{born}} C_{A}^{\mathrm{ew}}}{\left(C_{V}^{\mathrm{born}}\right)^{2}+\left(C_{A}^{\mathrm{born}}\right)^{2}},
$$

where all coefficients can be taken at $\nu=1$.

\section{MATCHING PROCEDURE AND RESULTS}

The NNLL order electroweak matching conditions for the Wilson coefficients of the effective theory operators in Eqs. (2) are obtained from the one-loop electroweak corrections to the Standard Model amplitude for $e^{+} e^{-} \rightarrow t \bar{t}$ for on-shell external top quarks in the limit $\sqrt{s} \rightarrow 2 m_{t}$ (i.e. $v \rightarrow 0$ ), where the top quarks are at rest. For simplicity the electron mass is neglected except for the self energy corrections, the CKM matrix is considered to be the unit matrix and the bottom quark mass is neglected in the $\gamma t \bar{t}$ and $Z t \bar{t}$ vertex corrections (including the top quark wave function renormalization). If QED corrections are neglected, 
there are no contributions singular in $v$ that are associated with nontrivial NRQCD matrix elements and all contributions need to be absorbed directly into the effective theory matching conditions. Neglecting pure QED corrections the Standard Model amplitude can be written in the form

$$
\mathcal{A}=i \frac{\alpha \pi}{m_{t}^{2}}\left[\bar{v}_{e^{+}}\left(k^{\prime}\right) \gamma^{\mu}\left(h_{R}^{\mathrm{ew}} \omega_{+}+h_{L}^{\mathrm{ew}} \omega_{-}\right) u_{e^{-}}(k)\right]\left[\bar{u}_{t}(p) \gamma_{\mu} v_{\bar{t}}(p)\right]
$$

where $\omega_{ \pm} \equiv \frac{1}{2}\left(1 \pm \gamma_{5}\right)$ and $k+k^{\prime}=2 p=\left(2 m_{t}, 0\right)$. The real parts of the NNLL electroweak matching conditions then read

$$
\begin{aligned}
& C_{V}^{\mathrm{ew}}(\nu=1)=\frac{\alpha \pi}{2 m_{t}^{2}} \operatorname{Re}\left[h_{R}^{\mathrm{ew}}+h_{L}^{\mathrm{ew}}\right], \\
& C_{A}^{\mathrm{ew}}(\nu=1)=\frac{\alpha \pi}{2 m_{t}^{2}} \operatorname{Re}\left[h_{R}^{\mathrm{ew}}-h_{L}^{\mathrm{ew}}\right] .
\end{aligned}
$$

To our knowledge the coefficients $h_{L, R}^{\mathrm{ew}}$ were first computed in Ref. [17] in Feynman gauge using the on-shell scheme for the initial state $e^{+} e^{-}$pair, the final state $t \bar{t}$ pair and the intermediate $\mathrm{Z}$ boson, and employing the fine structure constant for the electromagnetic coupling $\alpha^{-1}=137.036$. We have determined the real parts of the coefficients $h_{L, R}$ in the same renormalization scheme by hand and, independently, using the automated packages FeynArts [23] and FormCalc 24] (except for the $Z \gamma$ box diagrams). For the presentation of our results we follow (with minimal modifications) the conventions and notations used in Ref. 17. Since all components of the coefficients $h_{L, R}$ were presented explicitly in Ref. 17] we discuss in detail only those elements where our results differ. For undefined symbols and variables used in the following discussion we refer the reader to Ref. [17].

The results for the coefficients $h_{L, R}^{\mathrm{ew}}$ can be cast into the form

$$
h_{L, R}^{\mathrm{ew}}=h_{L, R}^{\mathrm{SE}}+h_{L, R}^{e^{+} e^{-}}+h_{L, R}^{t \bar{t}}+h_{L, R}^{\mathrm{box}},
$$

where $\left(M^{2}=s=4 m_{t}^{2}\right)$

$$
\begin{aligned}
h_{L, R}^{\mathrm{SE}}= & Q_{e}\left(-\frac{\Pi_{R}^{A A}}{M^{2}}\right) Q_{t}+\beta_{L, R}^{e} \frac{M^{2}}{M^{2}-M_{Z}^{2}}\left(-\frac{\Pi_{R}^{Z Z}}{M^{2}-M_{Z}^{2}}\right) \frac{\beta_{R}^{t}+\beta_{L}^{t}}{2} \\
& -\left(Q_{e} \frac{\beta_{R}^{t}+\beta_{L}^{t}}{2}+\beta_{L, R}^{e} Q_{t}\right) \frac{\Pi_{R}^{Z A}}{M^{2}-M_{Z}^{2}} \\
h_{L, R}^{e^{+} e^{-}}= & F_{L, R}^{A} Q_{t}+F_{L, R}^{Z} \frac{M^{2}}{M^{2}-M_{Z}^{2}} \frac{\beta_{R}^{t}+\beta_{L}^{t}}{2} \\
h_{L, R}^{t \bar{t}}= & Q_{e} \frac{\alpha}{4 \pi} \sum a^{A}+\beta_{L, R}^{e} \frac{M^{2}}{M^{2}-M_{Z}^{2}} \frac{\alpha}{4 \pi} \sum a^{Z} \\
h_{L, R}^{\mathrm{box}}= & h_{L, R}^{W W}+h_{L, R}^{Z Z}+h_{L, R}^{Z \gamma}
\end{aligned}
$$

and

$$
\begin{aligned}
\sum a^{A, Z} \equiv & a^{A, Z}(W)+a^{A, Z}(W, W)+a^{A, Z}\left(\phi_{W}\right)+a^{A, Z}\left(\phi_{W}, \phi_{W}\right) \\
& +a^{A, Z}\left(W, \phi_{W}\right)+a^{A, Z}(Z)+a^{A, Z}\left(\phi_{Z}\right)+a^{A, Z}(H) \\
& +a^{A, Z}(Z, H)+a^{A, Z}\left(\phi_{Z}, H\right)+a_{\mathrm{ct}}^{A, Z}
\end{aligned}
$$


and

$$
\beta_{R}^{f}=-\frac{s_{w}^{2} Q_{f}}{s_{w} c_{w}}, \quad \beta_{L}^{f}=\frac{t_{3, f}-s_{w}^{2} Q_{f}}{s_{w} c_{w}},
$$

$t_{3, f}$ being the third component of the weak isospin of fermion $f$.

The terms $h_{L, R}^{\mathrm{SE}}$ describe the self energy corrections where $\Pi_{R}^{i}, i=A A, Z Z, Z A$, are the renormalized transverse photon and Z self energy corrections and the photon-Z mixing correction. Compared to the expressions given in Ref. [17] our result does not sum the photon and $\mathrm{Z}$ boson self energy corrections into the denominator of the respective propagators since the resulting higher order corrections are beyond NNLL order and because at the $t \bar{t}$ threshold the intermediate photon and Z boson are far off-shell. The terms $h_{L, R}^{e^{+} e^{-}}$describe the corrections to the $e^{+} e^{-}$vertex, where $F_{L, R}^{i}, i=A, Z$, are the vertex corrections to the left- and right-handed $e^{+} e^{-} \gamma$ and $e^{+} e^{-} Z$ vertices. The terms $h_{L, R}^{t \bar{t}}$ refer to the corrections to the $t \bar{t}$ vertices, where the coefficients $a^{A, Z}$ correspond to the corrections to the $\gamma t \bar{t}$ and $Z t \bar{t}$ vertices, respectively. The arguments of the various terms in Eq. (20) indicate the virtual bosons that are being exchanged in the various triangle diagrams, e.g. $a^{A, Z}(W, W)$ refers to the vertex diagrams with exactly two internal $\mathrm{W}$ lines and $a^{A, Z}\left(\phi_{Z}\right)$ to those where the only internal boson is the neutral pseudo-Goldstone boson. Note that $a^{A, Z}\left(W, \phi_{W}\right), a^{A, Z}\left(\phi_{Z}, H\right)$ and $a^{A}(Z, H)$ are identically zero in Feynman gauge. Finally, the terms $h_{L, R}^{\text {box }}$ describe the contributions from the $W W, Z Z$ and $Z \gamma$ box diagrams. We note that the $Z \gamma$ box diagrams are infrared-finite for the $t \bar{t}$ pair being at rest.

Our results agree with those given in Ref. [17] except for the following points: ${ }^{3}$

1. For the corrections to the left-handed $e^{+} e^{-} \gamma$ and $e^{+} e^{-} Z$ vertices the coefficients $F_{L}^{A, Z}$ read

$$
\begin{aligned}
& F_{L}^{A}=\frac{\alpha}{4 \pi}\left(Q_{e}\left(\beta_{L}^{e}\right)^{2} \rho\left(q, M_{Z}\right)-\frac{t_{3}^{e}}{s_{w}^{2}} \Lambda\left(q, M_{W}\right)\right) \\
& F_{L}^{Z}=\frac{\alpha}{4 \pi}\left(\left(\beta_{L}^{e}\right)^{3} \rho\left(q, M_{Z}\right)+\left(\beta_{L}^{e}-2 t_{3}^{e} \frac{c_{w}}{s_{w}}\right) \frac{1}{2 s_{w}^{2}} \rho\left(q, M_{W}\right)-\frac{c_{w}}{s_{w}} \frac{t_{3}^{e}}{s_{w}^{2}} \Lambda\left(q, M_{W}\right)\right)
\end{aligned}
$$

We agree with the result for the function $\rho$ in [17], but for the function $\Lambda$ we find

$$
\Lambda\left(q, M_{W}\right)=-\frac{5}{2}+\frac{2}{u}-\left(1+\frac{2}{u}\right) l \sqrt{1-\frac{4}{u}}-\left(1+\frac{1}{2 u}\right) \frac{4}{u} l^{2},
$$

where

$$
l \equiv \ln \frac{\sqrt{1-4 / u}+1+i \epsilon}{\sqrt{1-4 / u}-1+i \epsilon}=\ln \frac{1+\sqrt{1-4 / u}}{1-\sqrt{1-4 / u}}-i \pi, \quad u \equiv \frac{M^{2}}{M_{W}^{2}} .
$$

For $\Delta^{\text {ew }}$, which describes the corrections to the total cross section from the real parts of the NNLL order electroweak matching conditions, this leads to an absolute shift by +0.012 with respect to the results in Ref. [17].

\footnotetext{
${ }^{3}$ We acknowledge that the discrepancies 2) and 3) were confirmed to us by [25].
} 
2. For the corrections to the $t \bar{t}$ vertices coming from the exchange of the neutral physical Higgs boson we find the result

$$
\begin{aligned}
a(H)= & \left(\begin{array}{c}
-Q_{t} \\
-\frac{1}{2}\left(\beta_{R}^{t}+\beta_{L}^{t}\right)
\end{array}\right) \frac{1}{s_{w}^{2}} \frac{M^{2}}{48 M_{W}^{2}}\left\{1+\left(3 \frac{M^{2}}{M_{H}^{2}}-1\right) B_{0}\left(\frac{1}{2} P, M_{H}, \frac{1}{2} M\right)\right. \\
& \left.+B_{1}\left(\frac{1}{2} P, M_{H}, \frac{1}{2} M\right)-3 \frac{M^{2}}{M_{H}^{2}} B_{0}\left(P, \frac{1}{2} M, \frac{1}{2} M\right)\right\},
\end{aligned}
$$

where the upper component refers to the photon and the lower to the $\mathrm{Z}$ vertex, respectively. Our results are consistent with the ones in Refs. 18, 26], but differ from those in Ref. [17] in the argument of the last $B_{0}$ function. Furthermore the counterterm contributions read

$$
\begin{aligned}
& a_{\mathrm{ct}}^{A}=\frac{2 \pi}{\alpha}\left(Q_{t}\left(\delta Z_{R}+\delta Z_{L}\right)+2 t_{3}^{t} \delta k\right), \\
& a_{\mathrm{ct}}^{Z}=\frac{2 \pi}{\alpha}\left(\left(\beta_{R}^{t} \delta Z_{R}+\beta_{L}^{t} \delta Z_{L}\right)+2 \frac{c_{w}}{s_{w}} t_{3}^{t} \delta k\right) .
\end{aligned}
$$

For the left- and right-handed wave function renormalization terms we have the expression

$$
\delta Z_{L, R}=A_{L, R}+m_{t}^{2}\left(A_{R}^{\prime}+A_{L}^{\prime}-2 C^{\prime}\right),
$$

where $C^{\prime} \equiv \frac{d C}{d p^{2}}\left(p^{2}=m_{t}^{2}\right)$. For the function $C$ we find

$$
\begin{aligned}
C= & \frac{\alpha}{4 \pi s_{w}^{2}}\left\{2 s_{w}^{2} \beta_{R}^{t} \beta_{L}^{t}\left(1-2 B_{0}\left(p, m_{t}, M_{Z}\right)\right)+\frac{m_{t}^{2}}{4 M_{W}^{2}}\left(B_{0}\left(p, m_{t}, M_{H}\right)\right.\right. \\
& \left.\left.-B_{0}\left(p, m_{t}, M_{Z}\right)\right)\right\},
\end{aligned}
$$

which is consistent with Ref. [18], but differs from Ref. [17], where the $B_{1}$ function was obtained instead of the first $B_{0}$ function. For $\Delta^{\text {ew }}$ these changes lead to an absolute shift by +0.076 with respect to the results in Ref. [17] for $m_{H}=130 \mathrm{GeV}$.

3. For the corrections from the $Z \gamma$ box diagrams we find the result

$$
\begin{aligned}
h_{R}^{Z \gamma} & =\frac{\alpha}{4 \pi}\left(-\beta_{R}^{e}\right)\left(\beta_{R}^{t}-\beta_{L}^{t}\right) Q_{e} Q_{t} 2 F^{Z \gamma}, \\
h_{L}^{Z \gamma} & =\frac{\alpha}{4 \pi}\left(\beta_{L}^{e}\right)\left(\beta_{R}^{t}-\beta_{L}^{t}\right) Q_{e} Q_{t} 2 F^{Z \gamma},
\end{aligned}
$$

where $\left(\tilde{u} \equiv \frac{M_{Z}^{2}}{M^{2}}\right)$

$$
\begin{aligned}
F^{Z \gamma}= & -\frac{1}{\tilde{u}-1}\left(B_{0}\left(\frac{1}{2} P, \frac{1}{2} M, 0\right)+B_{0}\left(\frac{1}{2} P, \frac{1}{2} M, M_{Z}\right)-2 B_{0}\left(P, 0, M_{Z}\right)\right) \\
& +\left(B_{0}\left(P, 0, M_{Z}\right)-B_{0}\left(l, 0, \frac{1}{2} M\right)\right)+(\tilde{u}-1) M^{2} C_{0}\left(l, \frac{1}{2} P, 0, \frac{1}{2} M, M_{Z}\right) .
\end{aligned}
$$

The expression for $F^{Z \gamma}$ agrees with the one in Ref. [27], but differs from the one in Ref. 17] by an additional minus sign in front of the first term. We note that the same error is also contained in the analysis of Ref. [18]. For $\Delta^{\text {ew }}$ the changes lead to an absolute shift by +0.004 with respect to the results in Ref. [17]. 
At this point we also take the opportunity to point out a number of discrepancies we find to Ref. [18]. (For the definition of the various undefined variables we refer the reader to Ref. [18].) For the WW box contribution we find

$$
F^{W W}(r)=\frac{2}{r-1}\left[l_{1}-l_{3}+\frac{1}{4}\left(f_{2}-r f_{1}\right)\right]
$$

where the function $f_{1}$ is defined by

$$
f_{1}(r)=-\left(\ln \frac{1+\beta}{1-\beta}-i \pi\right)^{2}, \quad \beta \equiv \sqrt{1-r} .
$$

This differs from [18] (in the published version) by the factor $r$ multiplying the function $f_{1}$ and the global minus sign in the definition of $f_{1}$. For the contribution of the $Z \gamma$ box diagrams we find the expression

$$
F^{Z \gamma}(r)=+2 \ln r+\ln \frac{4}{r}-\left(-1-\frac{r}{4}\right)\left(\ln \left(\frac{4}{r}-1\right)-i \pi\right)-\frac{4}{4-r} l_{4}+\left(\frac{r}{4}-1\right) f_{3} .
$$

For the $t \bar{t}$ vertex correction $a_{Z H}^{R}$, which corresponds to the triangle diagram containing a Z boson and a physical neutral Higgs boson, we find the expression

$$
\begin{aligned}
a_{Z H}^{R}\left(r_{H}, r_{Z}\right)= & \left(\begin{array}{l}
0 \\
1
\end{array}\right) \frac{\beta_{L}^{t}+\beta_{R}^{t}}{4 c_{w}^{2}}\left\{\frac{r_{H}+r_{Z}}{8} \ln \frac{r_{H}}{r_{Z}}+\frac{1}{r_{Z}+r_{H}-4}\left[-\left(4-r_{H}+r_{Z}\right) l_{5}+\left(4-r_{H}\right)\right.\right. \\
& \left.\left.\times\left[\left(1-\frac{r_{H}}{2}\right) \frac{1}{2} \ln r_{H} r_{Z}-l_{4}^{H}\right]+r_{Z}\left[\left(1-\frac{r_{Z}}{2}\right) \frac{1}{2} \ln r_{H} r_{Z}-l_{4}^{Z}\right]\right]\right\},
\end{aligned}
$$

which differs from the one in [18] by a minus sign in front of the function $l_{5}$.

\section{NUMERICAL ANALYSIS}

In this section we give a brief numerical discussion of the real parts of the NNLL electroweak matching conditions obtained in this work. In Tab. @ the numerical values for $C_{V, A}^{\text {born }}$ and $C_{V, A}^{\mathrm{ew}}$ are displayed for various values for the top and the Higgs masses, $\alpha^{-1}=137.036$ and

$$
\begin{array}{lll}
M_{W}=80.425 \mathrm{GeV}, & M_{Z}=91.1876 \mathrm{GeV}, & c_{w}^{2}=M_{W}^{2} / M_{Z}^{2}, \\
m_{e}=0.511 \mathrm{MeV}, & m_{\mu}=0.106 \mathrm{GeV}, & m_{\tau}=1.78 \mathrm{GeV}, \\
m_{u}=0.005 \mathrm{GeV}, & m_{d}=0.005 \mathrm{GeV}, & m_{s}=0.10 \mathrm{GeV}, \\
m_{c}=1.3 \mathrm{GeV}, & m_{b}=4.2 \mathrm{GeV} &
\end{array}
$$

for the gauge boson, the lepton and the quark masses. Note that the finite electron mass is applied in the calculation only for the self energy corrections.

The vector coefficients dominate for the tree level as well as for the one-loop coefficients. The one-loop corrections show a significant Higgs mass dependence and vary in the vector (axial-vector) case between $8.6 \%(-1.2 \%)$ and $5.6 \%(-2.6 \%)$ for Higgs masses between $115 \mathrm{GeV}$ and $1 \mathrm{TeV}$ and $m_{t}=172.5 \mathrm{GeV}$. 


\begin{tabular}{|l||r|r|r|r|r|r|r|r|}
\hline \multicolumn{1}{|l||}{$m_{t}(\mathrm{GeV})$} & \multicolumn{4}{c|}{170} & \multicolumn{1}{c|}{175} \\
\hline$m_{H}(\mathrm{GeV})$ & \multicolumn{1}{|c|}{115} & 150 & 200 & 1000 & \multicolumn{1}{c|}{115} & 150 & 200 & 1000 \\
\hline \hline$C_{V}^{\text {born }}\left(10^{-7} \mathrm{GeV}^{-2}\right)$ & -5.429 & & & & -5.123 & & & \\
\hline$C_{A}^{\text {born }}\left(10^{-7} \mathrm{GeV}^{-2}\right)$ & 1.260 & & & & 1.184 & & & \\
\hline$C_{V}^{\text {ew }}\left(10^{-8} \mathrm{GeV}^{-2}\right)$ & -4.562 & -4.073 & -3.702 & -3.060 & -4.460 & -3.951 & -3.566 & -2.890 \\
\hline$C_{A}^{\text {ew }}\left(10^{-9} \mathrm{GeV}^{-2}\right)$ & -1.416 & -2.286 & -2.797 & -3.025 & -1.408 & -2.335 & -2.904 & -3.260 \\
\hline$C_{V}^{\text {ew }} / C_{V}^{\text {born }}$ & 0.0840 & 0.0750 & 0.0682 & 0.0564 & 0.0871 & 0.0771 & 0.0696 & 0.0564 \\
\hline$C_{A}^{\text {ew }} / C_{A}^{\text {born }}$ & -0.0112 & -0.0181 & -0.0222 & -0.0240 & -0.0119 & -0.0197 & -0.0245 & -0.0275 \\
\hline \hline
\end{tabular}

TABLE I: Numerical values for the tree level and real one-loop electroweak matching conditions $C_{V, A}^{\text {born }}$ and $C_{V, A}^{\mathrm{ew}}$, respectively, and the $C_{V, A}^{\mathrm{ew}} / C_{V, A}^{\mathrm{born}}$ ratios for various values for the top and the Higgs masses, $\alpha^{-1}=137.036$ and the values given in Eq. (38). The coefficients $C_{V, A}^{\text {born }}$ do not depend on the Higgs mass.

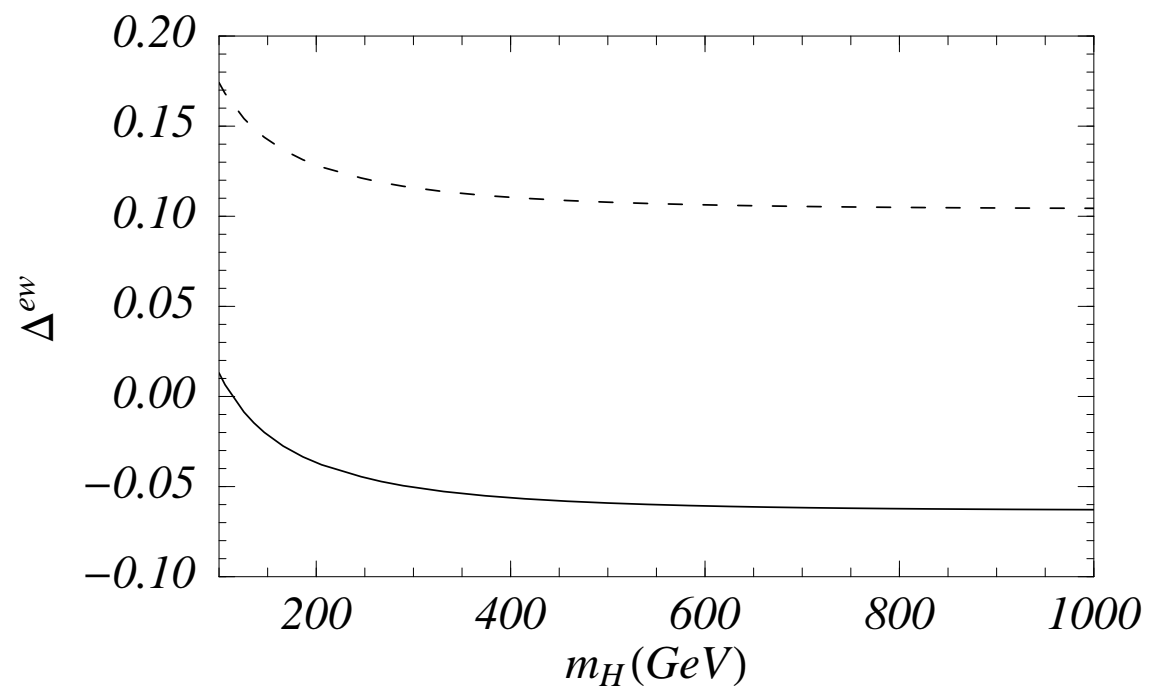

FIG. 1: The correction $\Delta^{\text {ew }}$ as a function of $m_{H}$ for $m_{t}=172.5 \mathrm{GeV}$ and the values given in Eq. (38) for $\alpha^{-1}=137.036$ (dashed curve) and in the scheme for the electromagnetic coupling defined in Eq. (39) (solid curve).

A more transparent view on the impact of the real electroweak one-loop corrections on the predictions of the total $t \bar{t}$ threshold cross section can be gained by considering the quantity $\Delta^{\text {ew }}$ defined in Eq. (12). In Fig. 1 the dashed line represents $\Delta^{\text {ew }}$ as a function of the Higgs mass for $m_{t}=172.5 \mathrm{GeV}$ and adopting the previous choices for the other parameters. For $m_{H}=(115,150,200,1000) \mathrm{GeV}$ we have $\Delta^{\mathrm{ew}}=(0.161,0.142,0.128,0.104)$. The Higgs mass dependence is rather strong for small $m_{H}$ and drops quickly close to the decoupling limit for increasing $m_{H}$. The strong Higgs mass dependence for small Higgs masses originates from the fact that for a light Higgs boson the dominant effects of the virtual Higgs exchange between the $t \bar{t}$ pair could be described in NRQCD by a Yukawa potential that leads to a singularity $\propto m_{t} / m_{H}$ for $m_{H} \rightarrow 0$ (see e.g. Refs. [18, 26, 28]). In an approach where the Higgs exchange 
would be accounted for in NRQCD through a Yukawa potential the electroweak one-loop matching conditions would need to be modified to account for the contributions caused by the Yukawa potential in the NRQCD matrix elements. If the Higgs boson is indeed very close to the lower LEP bound this would be a viable alternative to our approach where all virtual electroweak effects are encoded in the NRQCD Wilson coefficients.

The solid line in Fig. 1, finally, shows $\Delta^{\mathrm{ew}}$ for the same choice of parameters but adopting an $\overline{\mathrm{MS}}$ definition for the QED coupling at the scale $\mu=m_{t}$ that accounts for the leadinglogarithmic vacuum polarization effects due to the three charged leptons and the quarks below the top quark scale,

$$
\alpha^{n_{f}=8}(\mu)=\frac{\alpha}{1-\frac{\alpha}{3 \pi} \sum_{i=e, \mu, \tau} Q_{i}^{2} \ln \left(\frac{\mu^{2}}{m_{i}^{2}}\right)-\frac{\alpha}{3 \pi} \sum_{i=u, d, c, s, b} N_{c} Q_{i}^{2} \ln \left(\frac{\mu^{2}}{m_{i}^{2}}\right)} .
$$

In this scheme $\alpha^{n_{f}=8}\left(\mu=m_{t}\right)=1 / 125.926$ for $m_{t}=172.5 \mathrm{GeV}$ and the real NNLL electroweak matching conditions are modified,

$$
C_{V, A}^{\mathrm{ew}, \overline{\mathrm{MS}}}=C_{V, A}^{\mathrm{ew}}-C_{V, A}^{\mathrm{born}} \frac{\alpha^{n_{f}=8}(\mu)}{3 \pi}\left(\sum_{i=e, \mu, \tau} Q_{i}^{2} \ln \left(\frac{\mu^{2}}{m_{i}^{2}}\right)+\sum_{i=u, d, c, s, b} N_{c} Q_{i}^{2} \ln \left(\frac{\mu^{2}}{m_{i}^{2}}\right)\right) .
$$

This scheme is preferred to the one where the fine structure constant is used, since it leads to a substantially reduced dependence on the light fermion masses in $\Delta^{\mathrm{ew}}$. This is because most of the fermionic vacuum polarization effects are cancelled by the modification in Eq. (40). Moreover in this scheme $\Delta^{\mathrm{ew}}$ is by $17 \%$ smaller than in the one with the fine structure constant. For $m_{H}=(115,150,200,1000) \mathrm{GeV}$ we now have $\Delta^{\mathrm{ew}}=(-0.001,-0.021,-0.037,-0.063)$. The results show that concerning the real parts of the NNLL order electroweak matching corrections (up to the QED effects neglected in this work) the effects from virtual Higgs exchange are comparable to the other electroweak effects.

\section{CONCLUSION}

We have determined the real parts of the NNLL order (one-loop) electroweak matching conditions for the Wilson coefficients of the dominant NRQCD operators describing $t \bar{t}$ production close to threshold in $e^{+} e^{-}$annihilation. The results include the contributions of all electroweak one-loop effects that are integrated out when NRQCD is matched to the Standard Model except for pure QED corrections. The results are an important ingredient for a complete treatment of electroweak effects at NNLL order for top quark pair production

at a future Linear Collider. We have pointed out a number of discrepancies with respect to earlier work.

\section{Acknowledgments}

We would like to thank T. Teubner and J. H. Kühn for comments to the manuscript. 
[1] A. H. Hoang et al., in Eur. Phys. J. direct C 2, 1 (2000) arXiv:hep-ph/0001286.

[2] N. Brambilla et al., arXiv:hep-ph/0412158

[3] M. Battaglia et al., arXiv:hep-ph/0304132,

[4] M. Martinez and R. Miquel, Eur. Phys. J. C 27, 49 (2003) arXiv:hep-ph/0207315.

[5] A. A. Penin, V. A. Smirnov and M. Steinhauser, Nucl. Phys. B 716, 303 (2005) arXiv:hep-ph/0501042.

[6] M. Beneke, Y. Kiyo and K. Schuller, Nucl. Phys. B 714, 67 (2005) arXiv:hep-ph/0501289.

[7] A. H. Hoang, A. V. Manohar, I. W. Stewart and T. Teubner, Phys. Rev. Lett. 86, 1951 (2001) arXiv:hep-ph/0011254.

[8] A. H. Hoang, A. V. Manohar, I. W. Stewart and T. Teubner, Phys. Rev. D 65, 014014 (2001) arXiv:hep-ph/0107144.

[9] A. H. Hoang, Phys. Rev. D 69, 034009 (2004) arXiv:hep-ph/0307376.

[10] A. H. Hoang, Acta Phys. Polon. B 34, 4491 (2003) arXiv:hep-ph/0310301.

[11] S. T. Boogert and D. J. Miller, arXiv:hep-ex/0211021.

[12] D. Cinabro, arXiv:hep-ex/0005015.

[13] J. H. Kühn, in C80-05-29.6 Acta Phys. Polon. B 12, 347 (1981); I. I. Y. Bigi, Y. L. Dokshitzer, V. A. Khoze, J. H. Kühn and P. M. Zerwas, Phys. Lett. B 181, 157 (1986).

[14] V. S. Fadin and V. A. Khoze, JETP Lett. 46, 525 (1987).

[15] A. H. Hoang and C. J. Reisser, Phys. Rev. D 71, 074022 (2005) arXiv:hep-ph/0412258.

[16] A. H. Hoang and T. Teubner, Phys. Rev. D 60, 114027 (1999) arXiv:hep-ph/9904468.

[17] B. Grzadkowski, J. H. Kühn, P. Krawczyk and R. G. Stuart, Nucl. Phys. B 281, 18 (1987).

[18] R. J. Guth and J. H. Kühn, Nucl. Phys. B 368, 38 (1992).

[19] M. E. Luke, A. V. Manohar and I. Z. Rothstein, Phys. Rev. D 61, 074025 (2000) arXiv:hep-ph/9910209.

[20] A. H. Hoang and I. W. Stewart, Phys. Rev. D 67, 114020 (2003) arXiv:hep-ph/0209340.

[21] A. Pineda, Phys. Rev. D 66, 054022 (2002) arXiv:hep-ph/0110216.

[22] A. A. Penin, A. Pineda, V. A. Smirnov and M. Steinhauser, Nucl. Phys. B 699, 183 (2004) arXiv:hep-ph/0406175.

[23] T. Hahn, Comput. Phys. Commun. 140, 418 (2001) arXiv:hep-ph/0012260.

[24] T. Hahn and M. Perez-Victoria, Comput. Phys. Commun. 118, 153 (1999) arXiv:hep-ph/9807565.

[25] J. H. Kühn, private communication; T. Teubner, private communication.

[26] M. Jeżabek and J. H. Kühn, Phys. Lett. B 316, 360 (1993).

[27] R. G. Stuart, Comput. Phys. Commun. 48, 367 (1988).

[28] R. Harlander, M. Jeżabek and J. H. Kühn, Acta Phys. Polon. 27, 1781 (1996) arXiv:hep-ph/9506292. 